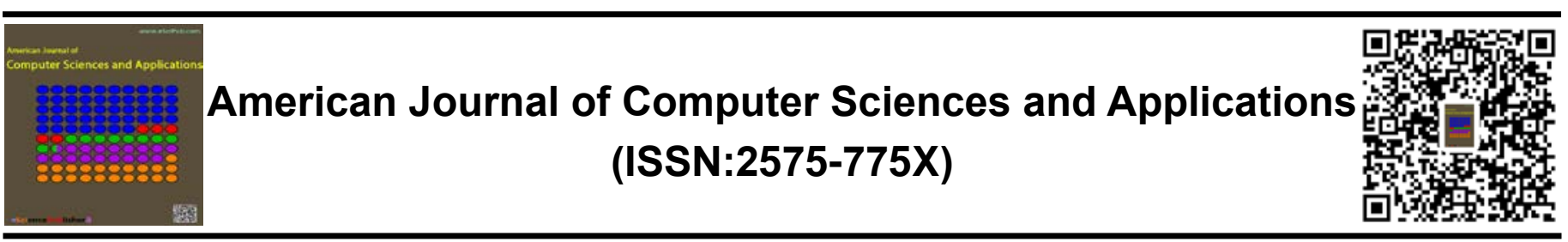

\title{
Compose Binary Matrices
}

\section{Dr. Ahmad Hamza Al Cheikha}

Dep. of Mathematical Science, College of Arts-science and Education, Ahlia Uni., Manama, Bahrain

\section{ABSTRACT}

Hadamard Matrices and M-Sequences (which formed a closed sets under the addition and with the corresponding null se*Correspondence to Author: quence formed additive groups and generated by feedback Dr. Ahmad Hamza Al Cheikha registers) are used widely at the forward links of communication channels to mix the information on connecting to and at the backward links of these channels to sift through this information Dep. of Mathematical Science, Colis transmitted to reach the receivers this information in correct form, specially in the pilot channels, the Sync channels, and the Traffic channel.

How to cite this article:

This research is useful to generate new sets of sequences (which Ahmad Hamza Al Cheikha. Comare also with the corresponding null sequence additive groups) by compose Hadamard matrices and M-sequences with the bigger lengths and the bigger minimum distance that assists to inpose Binary Matrices. American crease secrecy of these information and increase the possibility of correcting mistakes resulting in the channels of communication.

\section{eSciencePublisher}

Keywords: hadamard matrices, Walsh Sequences, M-sequenc- eSciPub LLC, Houston, TX USA. es, Additive group, Coefficient of Correlation, Orthogonal se- Website: http://escipub.com/ quences. 


\section{Introduction}

Ahmad Hamza Al Cheikha, AJCSA, 2017; 1:10
- M- Sequences: Let $k$ be a positive integer and $\lambda, \lambda_{0}, \lambda_{1}, \ldots, \lambda_{k-1}$ are elements in the field $F_{2}$, then the sequence $a_{0}, a_{1}, \ldots$ is called non homogeneous linear recurring sequence of order $k$ iff :

$a_{n+k}=\lambda_{k-1} a_{n+k-1}+\lambda_{k-2} a_{n+k-2}+\ldots+\lambda_{0} a_{n}+\lambda$,

$\lambda_{i} \in F_{2}, i=0,1, \ldots, k-1$

or $\quad a_{n+k}=\sum_{i=0}^{k-1} \lambda_{i} a_{n+i}+\lambda$

The elements $a_{0}, a_{1}, \ldots, a_{k-1}$ are called the initial values (or the vector $\left(a_{0}, a_{1}, \ldots, a_{k-1}\right)$ is called the initial vector). If $\lambda=0$ then the sequence $a_{0}, a_{1}, \ldots$ is called homogeneous linear recurring sequence (H. L. R. S.), except the zero-initial vector, and the polynomial

$f(x)=x^{k}+\lambda_{k-1} x^{k-1}+\ldots+\lambda_{1} x+\lambda_{0}$

is called the characteristic polynomial. In this study, we are limited to $\lambda_{0}=1$.

If the characteristic polynomial is prime then the sequence $a_{0}, a_{1}, \ldots$ is called $\mathrm{M}$-Sequence and

this sequence is periodic with period $n=2^{k}-1$, and each period contains $n_{1}=2^{k-1}-1$ of " $0 . s^{\text {" }}$ and $n_{2}=2^{k-1}$ of "1.s".

The set of all $n=2^{k}-1$ cyclic permutations of one period is closed under the addition by mod 2 and form an orthogonal set and any two different permutations contain $2^{k-1}$ of disagreements and $\left(2^{k-1}-1\right)$ of agreements, $f_{1}=\left(2^{k-2}-1\right)$ of the agreements are "0.s" and $f_{2}=2^{k-2}$ of the agreements are "1.s".

We can collect the all permutations of one period in one square matrix as following:

$A=\left[\begin{array}{cccc}a_{0} & a_{1} & \cdots & a_{n-1} \\ a_{n-1} & a_{0} & \cdots & a_{n-2} \\ \cdots & \cdots & \cdots & \cdots \\ a_{1} & \cdots & a_{n-1} & a_{0}\end{array}\right]$
Example 1: If $\alpha$ is a root of the prime polynomial $f(x)=x^{2}+x+1$ and generates $G F\left(2^{2}\right)$ and Suppose the Linear Binary Recurring Sequence be

$a_{n+2}=a_{n+1}+a_{n}$ or $a_{n+2}+a_{n+1}+a_{n}=0$

With the characteristic equation $x^{2}+x+1=0$ and the characteristic polynomial $f(x)=x^{2}+x+1$, which is a prime then the general solution of equation For the initial position: $a_{1}=1, a_{2}=0$ is given by: $a_{n}=\alpha \cdot \alpha^{n}+\alpha^{2} \cdot \alpha^{2 n}$, and the sequence is periodic with the period $2^{2}-1=3$ and $x_{1}=$ (101), by the cyclic permutations on $x_{1}$ we have $M_{3}=\left\{x_{1}, x_{2}, x_{3}\right\}$ where:

$x_{1}=(101), x_{2}=(110), x_{3}=(011)$, The first two digits in each sequence are the initial position

of the feedback register, and the set $M_{3}$ is an orthogonal set. The matrix of the all cyclic permutations is:

$A=\left[\begin{array}{lll}1 & 0 & 1 \\ 1 & 1 & 0 \\ 0 & 1 & 1\end{array}\right]$

$n=2^{2}-1=3 . \quad n_{1}=2^{2-1}-1=1, \quad n_{2}=2^{2-1}=2$,

$f_{1}=2^{2-1}-1=1, \quad f_{2}=2^{2-1}=2$

Example 2: The prime polynomial $f(x)=x^{3}+x+1$ is prime and generates $G F\left(2^{3}\right)$

Suppose the Linear Recurring Sequence be :

$a_{n+3}=a_{n+1}+a_{n}$ or $a_{n+3}+a_{n+1}+a_{n}=0$

The characteristic equation $x^{3}+x+1=0$ and the characteristic polynomial $f(x)=x^{3}+x+1$, which is a prime and generates $F_{2}$ and for the initial position: $a_{1}=1, a_{2}=0, a_{3}=0$, and the sequence is periodic with the period $2^{3}-1=7$ and $y_{1}=(1001011)$, by the cyclic permutations on $y_{1}$ we have 
$M_{7}=\left\{y_{1}, y_{2}, y_{3}, y_{4}, y_{5}, y_{6}, y_{7}\right\}$ where: $y_{2}=$

(1100101), $y_{3}=(1110010)$,

$y_{4}=(0111001), y_{5}=(1011100), y_{6}=(0101110)$,

$y_{7}=(0010111)$, the first three digits in each

sequence are the initial position of the feedback register, and the set $M_{7}$ is an orthogonal set. The matrix of the all cyclic permutations is:

$B=\left[\begin{array}{lllllll}1 & 0 & 0 & 1 & 0 & 1 & 1 \\ 1 & 1 & 0 & 0 & 1 & 0 & 1 \\ 1 & 1 & 1 & 0 & 0 & 1 & 0 \\ 0 & 1 & 1 & 1 & 0 & 0 & 1 \\ 1 & 0 & 1 & 1 & 1 & 0 & 0 \\ 0 & 1 & 0 & 1 & 1 & 1 & 0 \\ 0 & 0 & 1 & 0 & 1 & 1 & 1\end{array}\right]$

$m=2^{3}-1=7 . \quad m_{1}=2^{3-1}-1=3, \quad n_{2}=2^{3-1}=4$,

$g_{1}=2^{3-1}-1=3, \quad f_{2}=2^{3-1}=4$

[7], [10], [12], [15-18], [20], [27]

- Hadmard Matrices: Hadamard matrices seem such simple matrix structures: they are square, have entries +1 or -1 and have orthogonal row vectors and orthogonal column vectors.

A Hadamard matrix is invented by Sylvester (1867), 26 years before Hadamard (1893) considered them. The $n \times n$ Hadamard matrix $H_{n}$ must have $n(n-1) / 2$ of "- $1 . \mathrm{s}$ " and $n(n+1) / 2$ of "1.s". The binary representation of Hadamard matrix gets replaced each"1" by "0" and replaced each "-1" by"1"

Some basic properties of Hadamard matrices are given by following theorem:

Let $H_{h}$ be an Hadamard matrix of order $h$ then:

1. $H_{h} H_{h}^{t}=h I_{h}$, where $I_{h}$ is the identity matrix of order $h$;

2. $|\operatorname{det} H|=h^{\frac{1}{2} h}$;

3. $H_{h} H_{h}^{t}=H_{h}^{t} H_{h}$;

4. Hadamard matrices may be changed into other Hadamard matrices by permuting rows and columns and by multiplying rows and columns by -1 .
5. Matrices which can be obtained from one another by these methods are referred to as $\mathrm{H}$-equivalent (not all Hadamard matrices of the same order are $H$-equivalent).

6. Every Hadamard matrix is $\mathrm{H}$-equivalent to an Hadamard matrix which has every element of its first row and column equal +1 - matrices of this latter form are called normalized.

7. If $H_{4 n}$ is a normalized Hadamard matrix of order $4 n$, then every row (column), except the first, has $2 n$ minus ones and $2 n$ plus ones in each row (column), and the set these rows is called Walsh's sequences

8. The order of an Hadamad matrix is 1, 2, $4 n$, where $n$ is a positive integer.

(Sylvester) Let $H_{1}$ and $H_{2}$ be two Hadamard matrices of orders $h_{1}$ and $h_{2}$, then the

Kronecker product of $H_{1}$ and $H_{2}$ is an Hadamard matrix of order $h_{1} h_{2}$.

9. Standard Hadamard matrix $H_{h}$ have orthogonal rows (columns) vectors and each row (column) except the first row and first column contains $h / 2$ of " $1 . s$ " and $h / 2$ of " -1 " and $h / 2$ of disagreements and $h / 2$ of agreements $\mathrm{h} / 4$ of agreements are "1.s" and $\mathrm{h} / 4$ of agreements are "-1". [1-6], [8,9], [13,14], [19-26], [28,32].

The smallest examples are:

$H_{2^{0}}=[1], \quad H_{2^{1}}=\left[\begin{array}{cc}1 & 1 \\ 1 & -\end{array}\right], H_{2^{2}}=\left[\begin{array}{cccc}1 & 1 & 1 & 1 \\ 1 & - & 1 & - \\ 1 & 1 & - & - \\ 1 & - & - & 1\end{array}\right]$,

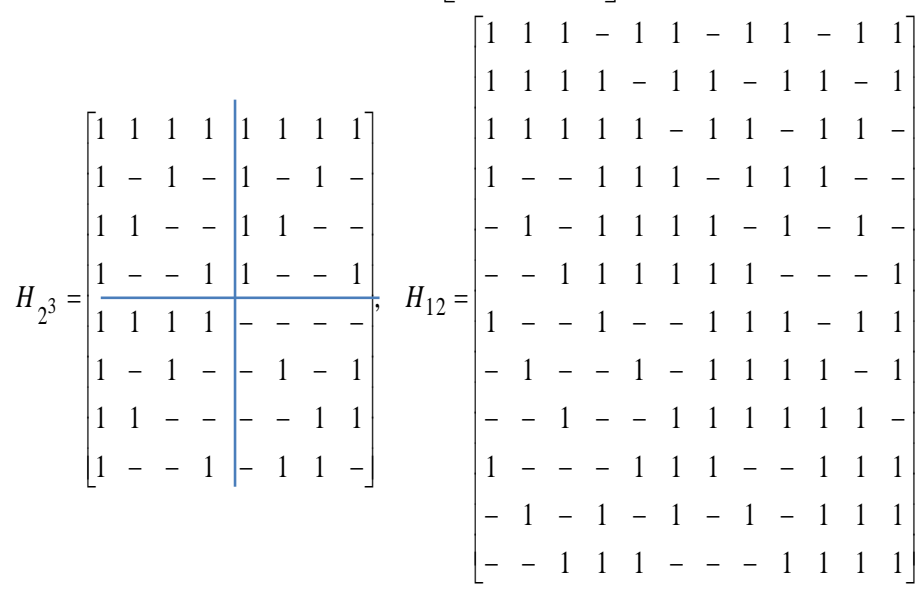


Research method and Material

Definition 1.The Ultimately Periodic Sequence $a_{0}, a_{1}, \ldots$.with the smallest period $r$ is called a periodic iff: $a_{n+r}=a_{n} ; n=0,1, \ldots[1,2],[12]$, [15-18], [20-27]

Definition 2. The complement of the binary vector $X=\left(x_{1}, x_{2}, \ldots, x_{n}\right), x_{i} \in F_{2}\{0,1\}$ is the vector $\bar{X}=\left(\overline{x_{1}}, \overline{x_{2}}, \ldots, \overline{x_{n}}\right)$, where:

$\overline{x_{i}}=\left\{\begin{array}{lll}1 & \text { if } & x_{i}=0 \\ 0 & \text { if } & x_{i}=1\end{array} .[1,2]\right.$

Definition 3. Suppose $x=\left(x_{0}, x_{1}, \ldots, x_{n-1}\right)$ and $y=\left(y_{0}, y_{1}, \ldots, y_{n-1}\right)$ are binary vectors of length $\mathrm{n}$ on $F_{2}=\{0,1\}$. The coefficient of correlations function of $x$ and $y$, denoted by $R_{x, y}$, is:

$$
R_{x, y}=\sum_{i=0}^{n-1}(-1)^{x_{i}+y_{i}}
$$

Where $x_{i}+y_{i}$ is computed mod 2. It is equal to the number of agreements components minus the number of disagreements corresponding to components or if $x_{i}, y_{i} \in\{1,-1\}$ (usually, replacing in binary vectors $x$ and $y$ each "1" by " 1 " and each " 0 " by "1") then

$$
R_{x, y}=\sum_{i=0}^{n-1} x_{i} y_{i},[1,2],[13-18]
$$

Definition 4. Any Periodic Sequence $a_{0}, a_{1}, \ldots$ over $F_{2}$ with prime characteristic polynomial is an orthogonal cyclic code and ideal auto correlation [1-2], [12], [18].

Definition 5. Suppose $x=\left(x_{0}, x_{1}, \ldots, x_{n-1}\right)$ and $y=\left(y_{0}, y_{1}, \ldots, y_{n-1}\right)$ are binary vectors of length $n$ on $G F(2)=\{0,1\}$, or components belong to $\{1,-1\}$, is said strictly orthogonal if $R_{x, y}=0$ and orthogonal if $R_{x, y} \in\{-1,0.1\}$ ). [1,2],[13-18]

Definition 6. Suppose $G$ is a set of binary vectors of length $n$ :

$$
G=\left\{\begin{array}{l}
X ; X=\left(x_{0}, x_{1}, \ldots, x_{n-1}\right), \\
x_{i} \in F_{2}=\{0,1\}, i=0,1, \ldots, n-1
\end{array}\right\}
$$

Let's $1^{*}=-1$ and $0^{*}=1$, The set $G$ is said to be strictly orthogonal if the following two conditions are satisfied:

1. $\forall X \in G, \sum_{i=0}^{n-1} x_{i}^{*}=0$, or $\left|R_{x, 0}\right|=0$.

2. $\forall X, Y \in G(X \neq Y), \sum_{i=0}^{n-1} x_{i}^{*} y_{i}^{*}=0$ or $\left|R_{x, y}\right|=0$.

That is, the absolute value of "the number of agreements minus the number of disagreements" is equal to zero, and orthogonal if the following two conditions are satisfied:

$$
\begin{aligned}
& \text { 1. } \forall X \in G, \sum_{i=0}^{n-1} x_{i}^{*} \leq 1, \text { or }\left|R_{x, 0}\right| \leq 1 . \\
& \text { 2. } \forall X, Y \in G(X \neq Y), \sum_{i=0}^{n-1} x_{i}^{*} y_{i}^{*} \leq 1 \text { or }\left|R_{x, y}\right| \leq 1 .
\end{aligned}
$$

That is, the absolute value of "the number of agreements minus the number of disagreements" is equal to zero. [1,2],[13-18]

Definition 7. The matrix $A=\left[a_{i j}\right]$ is called Hadamard matrix if it is a square matrix and each entry is equals 1 or "-" (where - denotes 1) with the property that if the size of $A$ is then $A^{T} A=A A^{T}=h I_{h}$, in the decimal counting system, and the distinct rows vectors are mutually orthogonal. [20-27]

Definition 8. If all entries of the first row and the first column in the Hadamard matrix are equal to " 1 " then the matrix is called standard Hadamard matrix.

Such matrices were first invented by Sylvester (1867) who observed that if $H$ is an Hadamard matrix, then:

$H=\left[\begin{array}{cc}H & H \\ H & -H\end{array}\right]$

Is also an Hadamard matrix. [3-12]

\section{Theorem 1.}

i. If $a_{0}, a_{1}, \ldots$ is a homogeneous linear recurring sequence of order $k$ in $F_{2}$, satisfies 
ii. (1) then this sequence is periodic

iii. If this sequence is homogeneous linear recurring sequence, periodic with the period $r$, and its characteristic polynomial $f(x)$ then $r \mid$ ord $f(x)$.

iv. If the polynomial $f(x)$ is primitive then the period of the sequence is $2^{k}-1$, and this sequence is called $\mathrm{M}$ - sequence. [7], [16], [17], [27]

Lema 2. (Sylvester (1867)): There is an Hadamard matrix of order $2^{t}$ for all nonnegative $t$. The matrices of order $2^{t}$ constructed using Sylvester's construction are usually Referred to as Sylvester-Hadamard matrices. [3-6], [13,15], Hadamard (1983) gave examples for a few small orders. [5-6],[8],[13-14],[22-23],[31-32]

\section{Results and Discussion}

First. Suppose $A$ and $B$ are tow square binary matrices of orders $n, m$ respectively, compose the matrix $A$ with the matrix $B$ or $A(B)$ is a the result of replacing each entry " 0 " in the matrix $A$ by the full matrix $B$ and replacing each "1" in the matrix $A$ by full $\bar{B}$ complement of the matrix $B$ and $A(B)$ is a square matrix of order n.m.

The study is similar when $A$ or $B$ is rectangular matrix.

Example 3. If :

$A=\left[\begin{array}{ll}1 & 1 \\ 1 & 0\end{array}\right]$ and $B=\left[\begin{array}{lll}1 & 1 & 0 \\ 0 & 1 & 1 \\ 1 & 0 & 1\end{array}\right]$

then: $\bar{B}=\left[\begin{array}{lll}0 & 0 & 1 \\ 1 & 0 & 0 \\ 0 & 1 & 0\end{array}\right]$

and: $A(B)=\left[\begin{array}{lll|lll}0 & 0 & 1 & 0 & 0 & 1 \\ 1 & 0 & 0 & 1 & 0 & 0 \\ 0 & 1 & 0 & 0 & 1 & 0 \\ \hline 0 & 0 & 1 & 1 & 1 & 0 \\ 1 & 0 & 0 & 0 & 1 & 1 \\ 0 & 1 & 0 & 1 & 0 & 1\end{array}\right]$

If: $A=\left[a_{i j}\right]_{n}=\left[\begin{array}{l}A_{1} \\ A_{2} \\ \vdots \\ A_{n}\end{array}\right]$,

$\boldsymbol{B}=\left[b_{k l}\right]_{m}=\left[\begin{array}{l}\boldsymbol{B}_{1} \\ \boldsymbol{B}_{2} \\ \vdots \\ \boldsymbol{B}_{m}\end{array}\right] \Rightarrow$

$A(B)=\left[\begin{array}{l}A_{1}\left(B_{1}\right) \\ A_{1}\left(B_{2}\right) \\ \cdots \\ A_{1}\left(B_{m}\right) \\ A_{2}\left(B_{1}\right) \\ A_{2}\left(B_{2}\right) \\ \cdots \\ A_{2}\left(B_{m}\right) \\ \vdots \\ A_{n}\left(B_{1}\right) \\ A_{n}\left(B_{2}\right) \\ \cdots \\ A_{n}\left(B_{m}\right)\end{array}\right]_{n \cdot m}$

And $A_{i j}=A_{i}\left(B_{j}\right)$ the result of compose the $i^{\text {th }}$ row in the matrix $A$ with the $j^{\text {th }}$ row of the matrix $B$.

If $A_{i}$ contains $n_{1}$ of "0.s" and $n_{2}$ of "1.s" then $\overline{A_{i}}$ contains $n_{1}$ of "1.s" and $n_{2}$ of "0.s" and if $B_{j}$ contains $m_{1}$ of " $0 . s$ " and $m_{2}$ of "1.s" then $\overline{B_{j}}$ contains $m_{1}$ of "1.s" and $m_{2}$ of "0.s" and $A_{i j}=A_{i}\left(B_{j}\right)$ contains $n_{1} m_{1}+n_{2} m_{2}$ of '0.s' and $n_{1} m_{2}+n_{2} m_{1}$ of "1.s" and $\bar{A}_{i j}=\bar{A}_{i}\left(\bar{B}_{j}\right)$ contains

The same number of '0.s' and of "1.s", from example 1.:

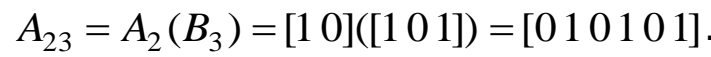

Our purpose now to study the following: From the information about two rows $A_{i_{1}}, A_{i_{2}}$ from the matrix $A$ and two rows $B_{j_{1}}, B_{j_{2}}$ from the matrix $B$ then what is the number of agreements and the number of disagreements between $A_{i_{1}}\left(B_{j_{1}}\right)$ and $A_{i_{2}}\left(B_{j_{2}}\right)$.

If there are two rows from the matrix $A$, for simplicity $A_{1}, A_{2}$ have: the same length 
$n=n_{1}+n_{2}$, where $n_{1}$ is the number of " $0 . \mathrm{s}$ " in $m=m_{1}+m_{2}$, where $m_{1}$ is the number of " $0 . \mathrm{s}$ " in each of them and $n_{2}$ is the number of "1.s" in each of them and the number of agreements each of them and $m_{2}$ is the number of "1.s" in between them is $f_{1}=r_{1}+r_{2}$ where $r_{1}$ of theme each of them and the number of agreements between them is $g_{1}=h_{1}+h_{2}$ where $h_{1}$ of theme are "0.s" and $r_{2}$ of theme are" $1 . s$ " such the are "0.s" and $h_{2}$ of

number of disagreements is theme are"1.s"such the number of $f_{2}=n-f_{1}=\left(n_{1}-r_{1}\right)+\left(n_{2}-r_{2}\right)=\left[n-\left(r_{1}+r_{2}\right)\right.$, by the disagreements

same way :

$g_{2}=m-g_{1}=\left(m_{1}-h_{1}\right)+\left(m_{2}-h_{2}\right)=\left[m-\left(h_{1}+h\right)_{2}\right]$.

If there are two rows from the matrix $B$, for Such the representation of the information as simplicity $B_{1}, B_{2}$ have: the same length following:

Representation 1: Compare $A_{1}\left(B_{1}\right)$ and $A_{2}\left(B_{2}\right)$

\begin{tabular}{|c|c|c|}
\hline \multirow[b]{3}{*}{$A_{1}\left(B_{1}\right) \Rightarrow$} & $n_{1}$ of "0.s" & $n_{2}$ of "1.s"' \\
\hline & $\left(n_{1}-r_{1}\right)$ & $\left(n_{2}-r_{2}\right)$ \\
\hline & 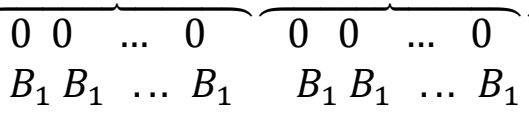 & $\begin{array}{lllllll}\begin{array}{llllll}1 & 1 & \ldots & 1 \\
\bar{B}_{1} \bar{B}_{1} & \ldots & \bar{B}_{1}\end{array} & l_{1 \bar{B}_{1}} \bar{B}_{1} & \ldots & \bar{B}_{1}\end{array}$ \\
\hline \multirow{3}{*}{$A_{2}\left(B_{2}\right) \Rightarrow$} & 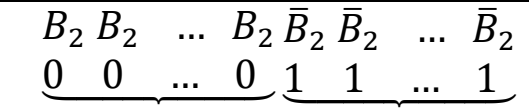 & 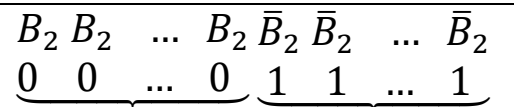 \\
\hline & $\left(n_{2}-r_{2}\right)$ & $\left(n_{1}-r_{1}\right)$ \\
\hline & $n_{1}$ of $0 . s^{\prime \prime}$ & $n_{2}$ of $1 . s^{\prime \prime}$ \\
\hline
\end{tabular}

- $\quad$ The number of agreements between $A_{1}$ and $A_{2}$ is: $f_{1}=r_{1}+r_{2}$.

- The number of disagreements between $A_{1}$ and $A_{2}$ is: $f_{2}=n-f_{1}=\left[n_{1}-\left(r_{1}+r_{2}\right)\right]$.

This representation showing a need to compare: $B_{1}$ and $B_{2}, B_{1}$ and $\bar{B}_{2}, \bar{B}_{1}$ and $B_{2}$ and $\bar{B}_{1}$ and $\bar{B}_{2}$,

a. Compare $B_{1}$ and $B_{2}$ : The Representation of the rows $B_{1}$ and $B_{2}$ of matrix $B$ is as following:

Representation 2: Compare $B_{1}$ and $B_{2}$

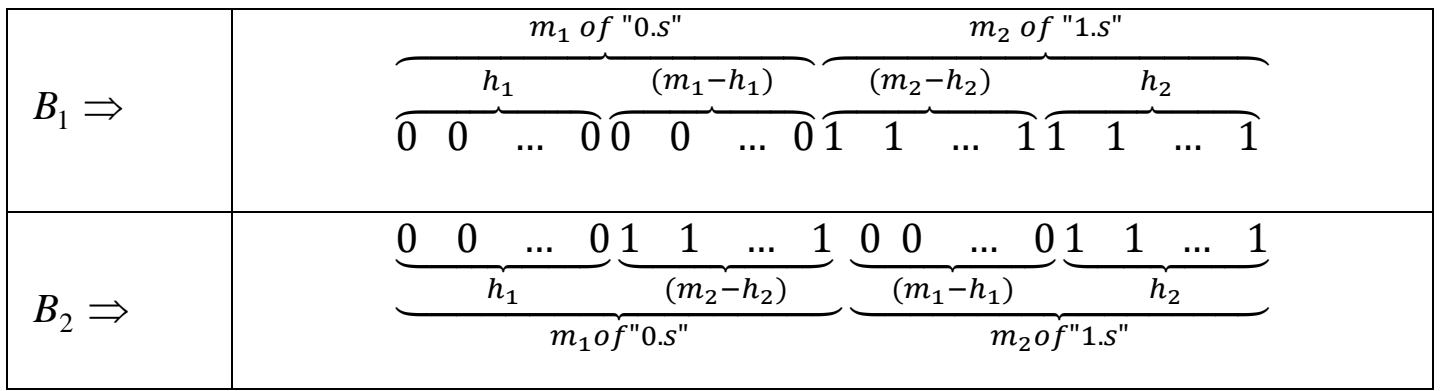

- The number of agreements between $B_{1}$ and $B_{2}$ is: $g_{1}=h_{1}+h_{2}$.

- The number of disagreements between $B_{1}$ and $B_{2}$ is: $g_{2}=m-g_{1}=\left[m-\left(h_{1}+h_{2}\right)\right]$.

b. Compare $B_{1}$ and $\bar{B}_{2}$ : The Representation of the rows $B_{1}$ and $\bar{B}_{2}$ of matrix $B$ is as following:

Representation 3: Compare $B_{1}$ and $\bar{B}_{2}$ 


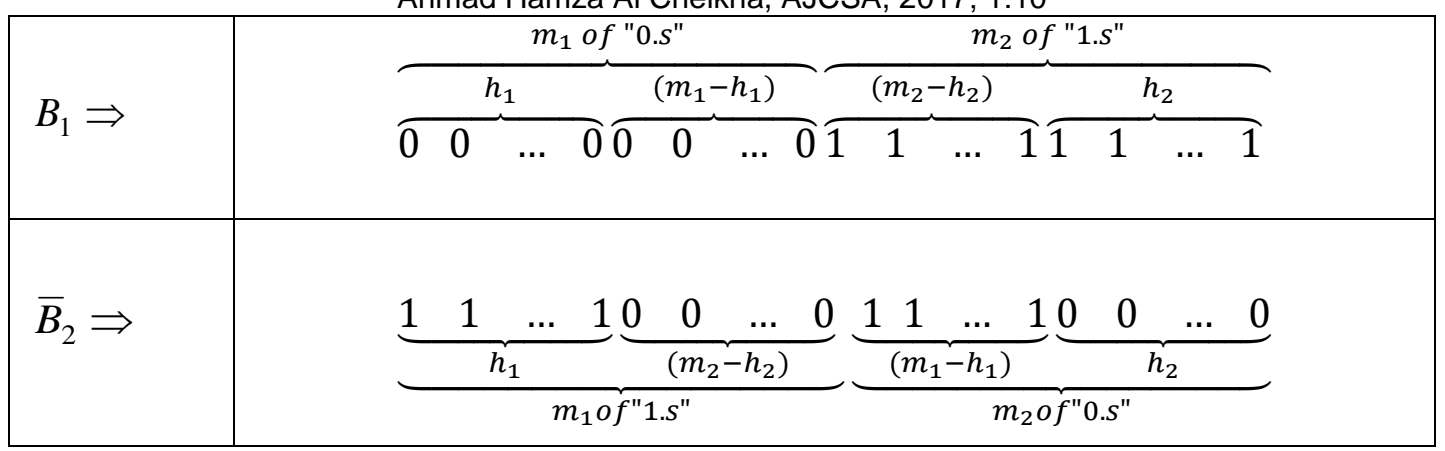

- The number of agreements between $B_{1}$ and $\bar{B}_{2}$ is: $g_{2}=m-g_{1}=\left[m-\left(h_{1}+h_{2}\right)\right]$.

- the number of Disagreements between $B_{1}$ and $\bar{B}_{2}$ is: $g_{1}=\left(h_{1}+h_{2}\right)$.

c. Compare $\bar{B}_{1}$ and $B_{2}$ : The Representation of the rows $B_{1}$ and $\bar{B}_{2}$ of matrix $B$ is as following:

Representation 4: Compare $\bar{B}_{1}$ and $B_{2}$

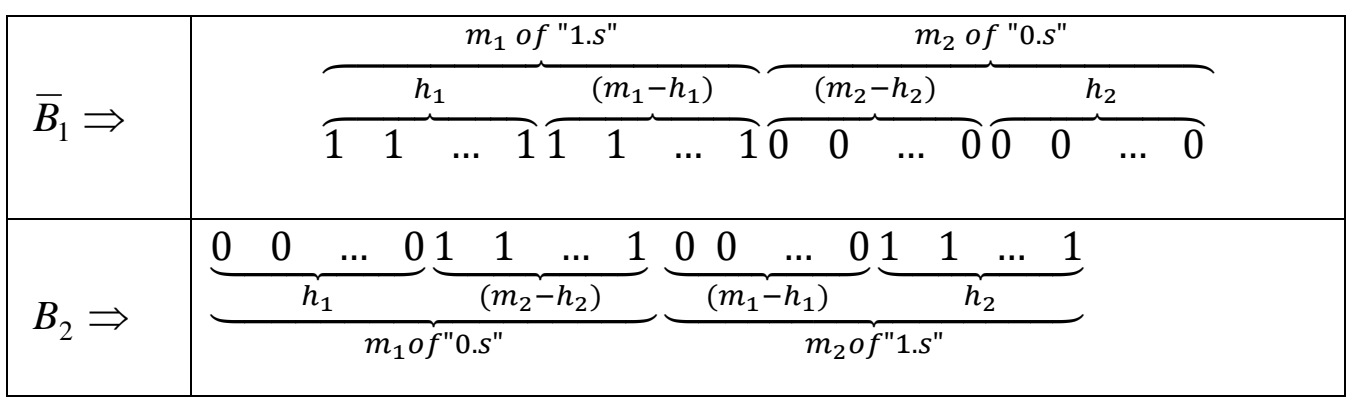

- The number of agreements between $\bar{B}_{1}$ and $B_{2}$ is: $g_{2}=m-g_{1}=\left[m-\left(h_{1}+h_{2}\right)\right]$.

The number of Disagreements between $\bar{B}_{1}$ and $B_{2}$ is: $g_{1}=h_{1}+h_{2}$.

d. Compare $\bar{B}_{1}$ and $\bar{B}_{2}$ : The Representation of the rows $\bar{B}_{1}$ and $\bar{B}_{2}$ of matrix $B$ is as following:

Representation 5: Compare $\bar{B}_{1}$ and $\bar{B}_{2}$

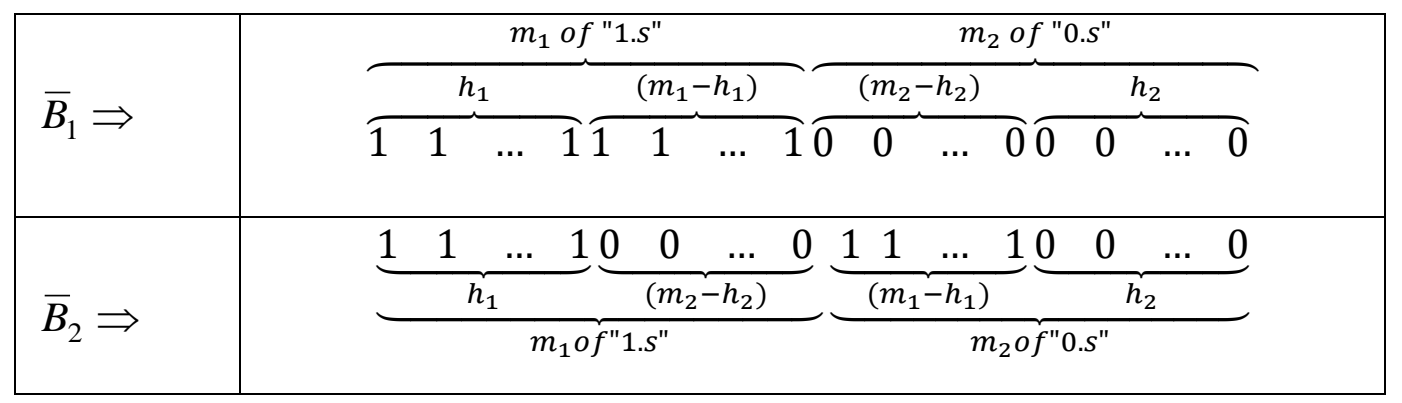

- The number of agreements between $\bar{B}_{1}$ and $\bar{B}_{2}$ is; $g_{1}=h_{1}+h_{2}$.

- The number of disagreements between $\bar{B}_{1}$ and $\bar{B}_{2}$ is: $g_{2}=\left[m-\left(h_{1}+h_{2}\right)\right]$.

First Step: For one row $A_{i}$ of the matrix $A$ and two different rows $B_{j}, B_{k}$ of the matrix $B$ then from the representations $(2,5)$ and from the following representation of $A_{i}, B_{j}, B_{k}$ : 
Representation 6: Compare $A_{i}\left(B_{j}\right)$ and $A_{i}\left(B_{k}\right)$

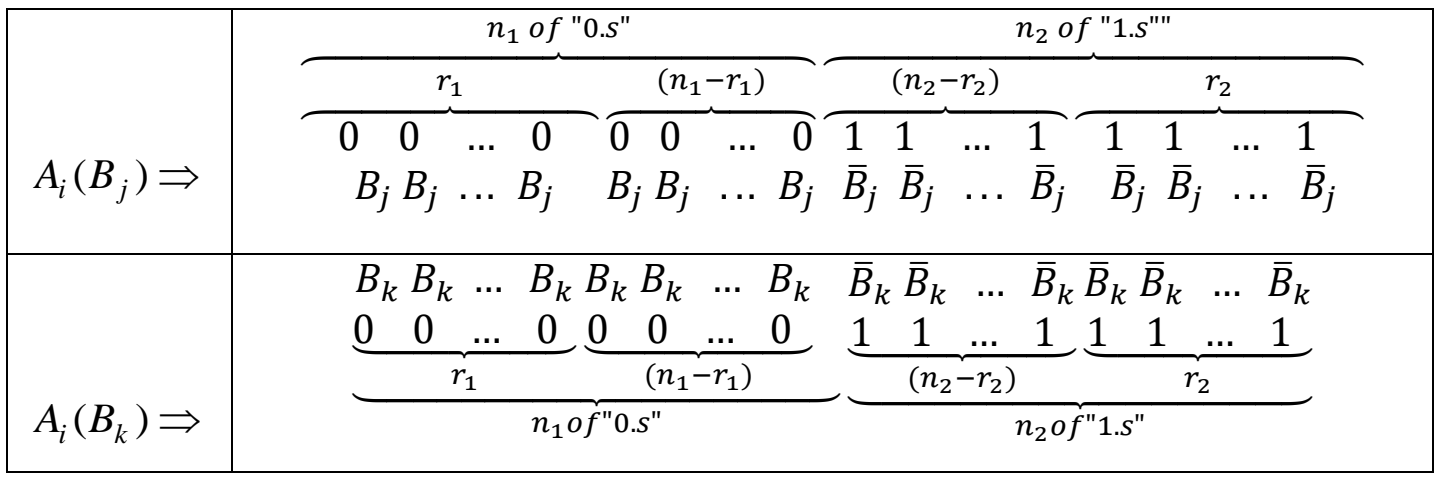

We have :

- The length of $A_{i}\left(B_{j}\right)$ and $A_{i}\left(B_{k}\right)$ is: n.m

- The number of " $0 . \mathrm{s}$ " in each of them is: $n_{1} m_{1}+n_{2} m_{2}$.

- The number of "1.s" in each of them is: $n_{1} m_{2}+n_{2} m_{1}$.
- The number of agreements is: $n g_{1}=n\left(h_{1}+h_{2}\right)$.

- The number of disagreements is: $n g_{2}=n\left[m-\left(h_{1}+h_{2}\right)\right]$.

- The difference $d$ between the disagreements and agreements is:

$d=\left|n g_{2}-n g_{1}\right|=\left|n\left[m-\left(h_{1}+h_{2}\right)\right]-n\left(h_{1}+h_{2}\right)\right|$ $d=\left|n\left[m-2\left(h_{1}+h_{2}\right)\right]\right|$

Second Step: For two different rows $A_{i}, A_{j}$ of the matrix $A$ and one row $B_{k}$ of the matrix $B$ and from the representations (2-5) and from the following representation of $A_{i}, A_{j}, B_{k}$ :

Representation 7: Compare $A_{i}\left(B_{k}\right)$ and $A_{j}\left(B_{k}\right)$

\begin{tabular}{|c|c|}
\hline & $n_{2}$ of "1.s"'" \\
\hline \multirow[b]{2}{*}{$A_{i}\left(B_{k}\right) \Rightarrow$} & $\left(n_{2}-r_{2}\right)$ \\
\hline & 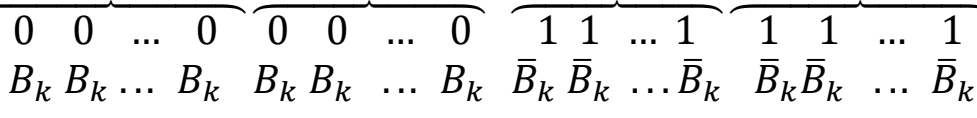 \\
\hline \multirow[b]{2}{*}{$A_{j}\left(B_{k}\right) \Rightarrow$} & 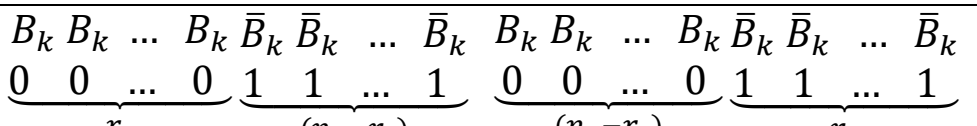 \\
\hline & $\begin{array}{ll}r_{1} & \left(n_{2}-r_{2}\right) \\
& n_{1} o f^{\prime \prime} 0 . s^{\prime \prime}\end{array}$ \\
\hline
\end{tabular}

We have :

- The length of $A_{i}\left(B_{k}\right)$ and $A_{j}\left(B_{k}\right)$ is: n.m

- The number of " $0 . s$ " in each of them is: $n_{1} m_{1}+n_{2} m_{2}$.

- The number of "1.s" in each of them is: $n_{1} m_{2}+n_{2} m_{1}$.
- The number of agreements is: $m f_{1}=m\left(r_{1}+r_{2}\right)$.

- The number of disagreements is: $m f_{2}=m\left[n-\left(r_{1}+r_{2}\right)\right]$.

- The difference $d$ between the disagreements and agreements is:

$d=\left|m f_{2}-m f_{1}\right|\left|m\left[n-\left(r_{1}+r_{2}\right)\right]-m\left(r_{1}+r_{2}\right) m\right|$ $d=\left|\left[n-2\left(r_{1}+r_{2}\right)\right] m\right|$ 
Third Step: For two different rows $A_{i}, A_{j}$ of the matrix $A$ and two different rows $B_{k}, B_{l}$ of the Matrix $B$ and from the representations (1-5) and from the following representation of $A_{i}, A_{j}, B_{k}, B_{l}:$

Representation 8: Compare $A_{i}\left(B_{k}\right)$ and $A_{j}\left(B_{l}\right)$

\begin{tabular}{|c|c|}
\hline \multirow[b]{3}{*}{$A_{i}\left(B_{k}\right) \Rightarrow$} & $n_{2}$ of "1.s"' \\
\hline & $\left(n_{2}-r_{2}\right)$ \\
\hline & 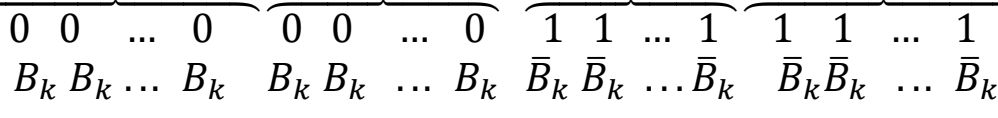 \\
\hline \multirow[b]{2}{*}{$A_{j}\left(B_{l}\right) \Rightarrow$} & 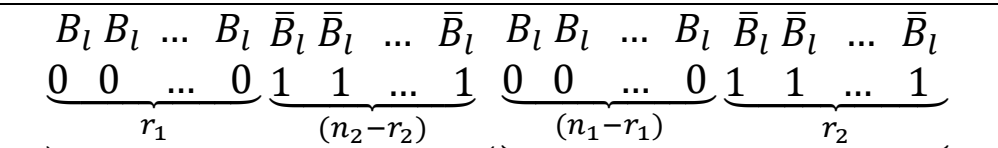 \\
\hline & $n_{n_{1} o f " 0 . s^{\prime \prime}}$ \\
\hline
\end{tabular}

We have:

- The number of agreements between $A_{i}$ and $A_{j}$ is: $f_{1}=\left(r_{1}+r_{2}\right)$.

- The number of disagreements between $A_{i}$ and $A_{j}$ is: $f_{2}=\left[n-\left(r_{1}+r_{2}\right)\right]$

- The number of agreements between $B_{k}$ and $B_{l}$ is: $g_{1}=\left(h_{1}+h_{2}\right)$.

- The number of disagreements between $B_{k}$ and $B_{1}$ is: $g_{2}=\left[m-\left(h_{1}+h_{2}\right)\right]$

- The length of $A_{i}\left(B_{k}\right)$ and $A_{j}\left(B_{l}\right)$ is: n.m

- The number of "0.s" in each of them is: $n_{1} m_{1}+n_{2} m_{2}$.

- The number of "1.s" in each of them is: $n_{1} m_{2}+n_{2} m_{1}$.
- The number of agreements between $A_{i}\left(B_{k}\right)$ and $A_{j}\left(B_{l}\right)$ is: $f_{1} g_{1}+f_{2} g_{2}$, or is: $\left(r_{1}+r_{2}\right)\left(h_{1}+h_{2}\right)+\left[n-\left(r_{1}+r_{2}\right)\right]\left[m-\left(h_{1}+h_{2}\right)\right]$

- The number of disagreements between $A_{i}\left(B_{k}\right)$ and $A_{j}\left(B_{l}\right)$ is: $f_{1} g_{2}+f_{2} g_{1}$, or is: $\left(r_{1}+r_{2}\right)\left[m-\left(h_{1}+h_{2}\right)\right]+\left[n-\left(r_{1}+r_{2}\right)\right]\left(h_{1}+h_{2}\right)$

- The difference $d$ between the agreements and disagreements of $A_{i}\left(B_{k}\right)$ and $A_{j}\left(B_{l}\right)$ is:

$d=\left(f_{1} g_{1}+f_{2} g_{2}\right)-\left(f_{1} g_{2}+f_{2} g_{1}\right)$

$d=\left(f_{1}-f_{2}\right)\left(g_{1}-g_{2}\right)$

Or:

$d=n m-2 n\left(h_{1}+h_{2}\right)-2 m\left(r_{1}+r_{2}\right)+4\left(r_{1}+r_{2}\right)\left(h_{1}+h_{2}\right)$

Example 4. Using binary representations of the standard Hadmard matrices:

$H_{2}=\left[\begin{array}{ll}0 & 0 \\ 0 & 1\end{array}\right], H_{4}=\left[\begin{array}{llll}0 & 0 & 0 & 0 \\ 0 & 1 & 0 & 1 \\ 0 & 0 & 1 & 1 \\ 0 & 1 & 1 & 0\end{array}\right], \ldots H_{8}=\left[\begin{array}{llllllll}0 & 0 & 0 & 0 & 0 & 0 & 0 & 0 \\ 0 & 1 & 0 & 1 & 0 & 1 & 0 & 1 \\ 0 & 0 & 1 & 1 & 0 & 0 & 1 & 1 \\ 0 & 1 & 1 & 0 & 0 & 1 & 1 & 0 \\ 0 & 0 & 0 & 0 & 1 & 1 & 1 & 1 \\ 0 & 1 & 0 & 1 & 1 & 0 & 1 & 0 \\ 0 & 0 & 1 & 1 & 1 & 1 & 0 & 0 \\ 0 & 1 & 1 & 0 & 1 & 0 & 0 & 1\end{array}\right], H_{12}=\left[\begin{array}{llllllllllll}0 & 0 & 0 & 0 & 0 & 0 & 0 & 0 & 0 & 0 & 0 & 0 \\ 0 & 0 & 0 & 1 & 1 & 0 & 1 & 1 & 0 & 1 & 1 & 0 \\ 0 & 0 & 0 & 1 & 0 & 1 & 1 & 0 & 1 & 1 & 0 & 1 \\ 0 & 1 & 1 & 1 & 0 & 0 & 0 & 0 & 0 & 1 & 1 & 1 \\ 0 & 1 & 0 & 0 & 1 & 1 & 0 & 0 & 1 & 1 & 1 & 0 \\ 0 & 0 & 1 & 0 & 1 & 1 & 0 & 1 & 0 & 1 & 0 & 1 \\ 0 & 1 & 1 & 1 & 1 & 1 & 1 & 0 & 0 & 0 & 0 & 0 \\ 0 & 1 & 0 & 1 & 1 & 0 & 0 & 1 & 1 & 0 & 0 & 1 \\ 0 & 0 & 1 & 1 & 0 & 1 & 0 & 1 & 1 & 0 & 1 & 0 \\ 0 & 1 & 1 & 0 & 0 & 0 & 1 & 1 & 1 & 1 & 0 & 0 \\ 0 & 1 & 0 & 0 & 0 & 1 & 1 & 1 & 0 & 0 & 1 & 1 \\ 0 & 0 & 1 & 0 & 1 & 0 & 1 & 0 & 1 & 0 & 1 & 1\end{array}\right], \cdots, H_{4 h_{1}}, \cdots$ 
The set of row of a standard Hadamard matrix form an additive group for addition by mod 2 and the set of this rows except the first row iii. Each row except the first row contains $4 h$ of also closed under the addition (Walsh's Sequences).

Except $\mathrm{H}_{2}$, First row contains only " $0 . \mathrm{s}$ " each of the other rows contains $2 h_{1}$ of " $0 . s$ ", $2 h_{1}$ of

"1.s", $2 h_{1}$ of disagreements and $2 h_{1}$ of agreements, $h_{1}$ of the agreements are "0.s" and the other

$h_{1}$ are "1.s".

i. Is very clear: $\mathrm{H} 2(\mathrm{H} 2)=\mathrm{H} 4$ is an Hadamard matrix.

ii. All entries of the first row in $H_{2}\left(H_{4 h}\right)$ are "O" and each other row contains $4 h$ of " $0 . s$ " and $4 h$ of "1.s" and orthogonal with the first row, the order of the matrix $\mathrm{H}_{2}\left(\mathrm{H}_{4 h}\right)$ is $8 h$.
$H_{2}\left(H_{4 h}\right)=\left[\begin{array}{ll}H_{4 h} & H_{4 h} \\ H_{4 h} & H_{4 h}\end{array}\right]$ "0.s", $4 h$ of " $1 . s$ " and any two different rows except the first contain $4 h$ of disagreements and $4 h$ of agreements (from third. The difference between the agreements and disagreements

is:

$\left.d=\left(f_{1}-f_{2}\right)\left(g_{1}-g_{2}\right)=(1-1)(2 h-2 h)=0\right)$

of the agreements are "0.s" and the other $2 h$ are "1.s" also because this set of rows is closed under the addition. Thus these two rows are orthogonal and the columns of $H_{2}\left(H_{4 h}\right)$ have the same properties of the rows and $H_{2}\left(H_{4 h}\right)$ is an Hadamard matrix. By the same way for $H_{4 h}\left(H_{2}\right)$ is an Hadamard matrix.

\section{Example 5.}

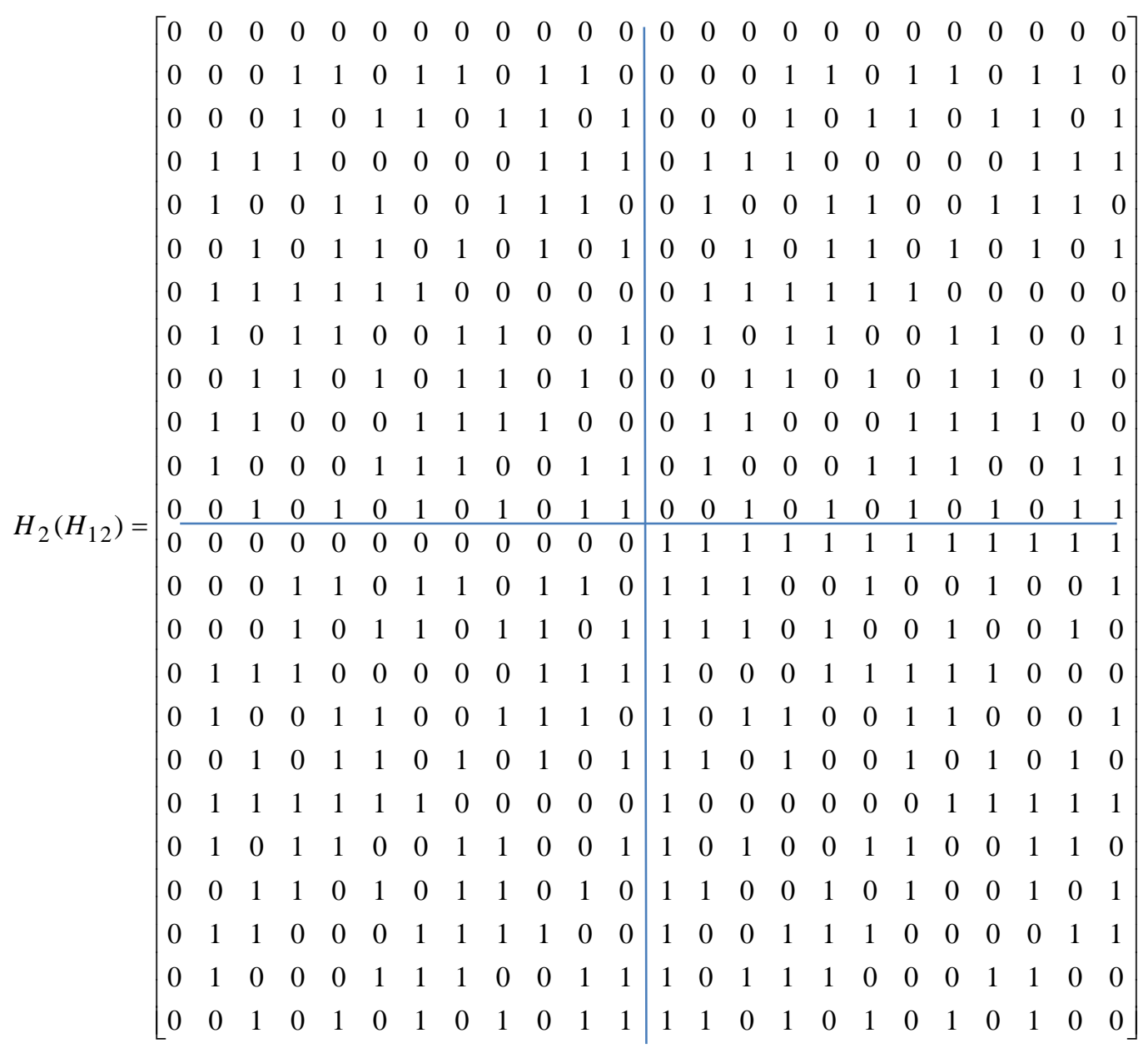


In this matrix, except the first row (column), each row (column) contains 12 of "0.s" , 12 of "1.s", 12 of disagreements and 12 of agreements, 6 of the agreements are "0" and 6 of agreements are "1.s"

iv. All entries of the first row in $H_{4 h_{1}}\left(H_{4 h_{2}}\right)$ are "0" and each other row contains $8 h_{1} h_{2}$ of "0.s" and $8 h_{1} h_{2}$ of "1.s" and orthogonal with the first row and the order of the matrix is: $16 h_{1} h_{2}$.

For any two different rows, except the first row

- The number of agreements between $A_{i}$ and $A_{j}$ is: $f_{1}=\left(h_{1}+h_{1}\right)=2 h_{1}$.

- The number of disagreements between $A_{i}$ and $A_{j}$ is: $f_{2}=\left[4 h_{1}-2 h_{1}\right]=2 h_{1}$

- The number of agreements between $B_{k}$ and $B_{1}$ is: $g_{1}=\left(h_{2}+h_{2}\right)=2 h_{2}$.

- The number of disagreements between $B_{k}$ and $B_{1}$ is: $g_{2}=\left[4 h_{2}-2 h_{2}\right]=2 h_{2}$

* In the composition $A_{i}\left(B_{j}\right)$ and $A_{i}\left(B_{k}\right)$ :

- The number of agreements is: $n g_{1}=4 h_{1}\left(2 h_{2}\right)=8 h_{1} h_{2}$.

- The number of disagreements is: $n g_{2}=4 h_{1}\left(2 h_{2}\right)=8 h_{1} h_{2}$.

- The difference $d$ between the disagreements and agreements is: $d=0$

Thus, second condition of the orthogonal is verified.

* In the composition $A_{i}\left(B_{k}\right)$ and $A_{j}\left(B_{k}\right)$ :

- The number of agreements $m f_{1}=4 h_{2}\left(2 h_{1}\right)=8 h_{1} h_{2}$.

- The number of disagreements is: $m f_{2}=4 h_{2}\left(2 h_{1}\right)$.

- The difference $d$ between the disagreements and agreements is: $d=0$

Thus, second condition of the orthogonal is verified.

* In the composition $A_{i}\left(B_{k}\right)$ and $A_{j}\left(B_{l}\right)$ :
- The number of agreements is:

$f_{1} g_{1}+f_{2} g_{2}=2 h_{1}\left(2 h_{2}\right)+2 h_{1}\left(2 h_{2}\right)=8 h_{1} h_{2}$.

- The number of disagreements is: $f_{1} g_{2}+f_{2} g_{1}=2 h_{1}\left(2 h_{2}\right)+2 h_{1}\left(2 h_{2}\right)=8 h_{1} h_{2}$.

- The difference $d$ between the disagreements and agreements is "0", or :

$d=\left|\left(2 h_{1}-2 h_{1}\right)\left(2 h_{2}-2 h_{2}\right)\right|=0$

- The columns of $H_{4 h_{1}}\left(H_{4 h_{2}}\right)$ have the same properties of the rows and $H_{4 h_{1}}\left(H_{4 h_{2}}\right)$ is an Hadamard matrix. By the same way for $H_{4 h_{2}}\left(H_{4 h_{1}}\right)$ is an Hadamard matrix.

Thus, second condition of the orthogonal is verified and rows of $A(B)$ except the first row form an orthogonal set

Second. Compose binary matrices is associative: We will prove that $A(B(C))=(A(B))(C)$ for any $A, \quad B, \quad C$ binary matrices.

Suppose:

$A=\left[a_{i j}\right]_{h_{1}}=\left[\begin{array}{l}A_{1} \\ A_{2} \\ \vdots \\ A_{h_{1}}\end{array}\right], B=\left[b_{k l}\right]_{h_{2}}=\left[\begin{array}{l}B_{1} \\ B_{2} \\ \vdots \\ B_{h_{2}}\end{array}\right], C=\left[c_{m n}\right]_{h_{3}}=\left[\begin{array}{l}C_{1} \\ C_{2} \\ \vdots \\ C_{h_{3}}\end{array}\right]$

From the left side:

i. $A(B(C))=\left\lfloor a_{i j}(B(C))\right\rfloor_{h_{1} \cdot h_{2} \cdot h_{3}}, i, j=1,2, \ldots, h_{1}$

- If $a_{i j}=0$ then:

From the left side:

$a_{i j}(B(C))=(B(C))=\left[\begin{array}{l}B_{1}(C) \\ B_{2}(C) \\ \ldots \\ B_{h_{2}}(C)\end{array}\right]=\left[\begin{array}{llll}b_{11}(C) & b_{12}(C) & \ldots \ldots \ldots & b_{1 h_{2}}(C) \\ b_{21}(C) & b_{22}(C) & \ldots \ldots \ldots & b_{2 h_{2}}(C) \\ \ldots \ldots \ldots \ldots \ldots \ldots \ldots \ldots & \ldots \ldots \ldots \\ b_{h_{21} 1}(C) & b_{h_{2} 2}(C) & \ldots & b_{h_{2} 1 h_{2}}(C)\end{array}\right]$

From the right side:

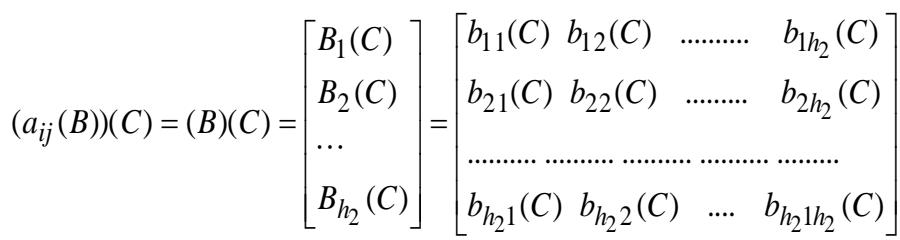

Thus: $A(B(C))=(A(B)(C)$

- If $a_{i j}=1$ then:

From the left side:

Http://escipub.com/american-journal-of-computer-sciences-and-applications/ 


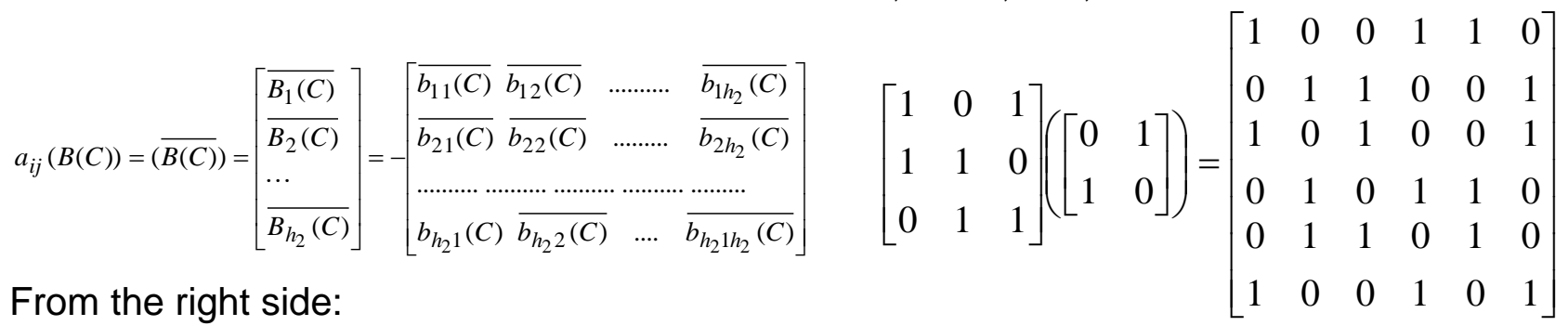

$$
\left(a_{i j}(B)\right)(C)=(\bar{B}(C))=\left[\begin{array}{l}
\bar{B}_{1}(C) \\
\bar{B}_{2}(C) \\
\ldots \\
\overline{B_{h}}(C)
\end{array}\right]=\left[\begin{array}{llll}
\overline{b_{11}}(C) & \overline{b_{12}}(C) & \ldots \ldots \ldots . & \overline{b_{1 h_{2}}}(C) \\
\overline{b_{21}}(C) & \overline{b_{22}}(C) & \ldots \ldots \ldots & \overline{b_{2 h_{2}}}(C) \\
\ldots \ldots \ldots \ldots \ldots \ldots \ldots \ldots \ldots \ldots \ldots \ldots \ldots \ldots \\
\overline{b_{h_{2} 1}}(C) & \overline{b_{h_{2} 2}}(C) & \ldots \ldots & \overline{b_{h_{2} 1 h_{2}}}(C)
\end{array}\right]
$$

Is $\bar{B}(C)=\overline{B(C)}$ ?

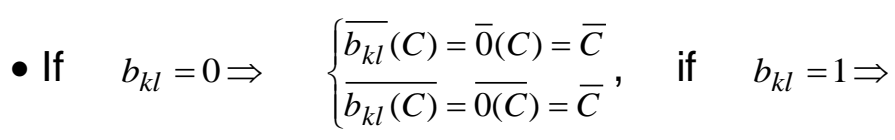

$$
\left\{\begin{array}{l}
\overline{b_{k l}}(C)=\overline{1}(C)=0(C)=C \\
\overline{b_{k l}(C)}=\overline{1(C)}=\overline{\bar{C}}=C
\end{array}\right.
$$

Thus $\bar{B}(C)=\overline{B(C)}$

Thus for any binary matrices $A, B, C: A(B(C))=$ $(A(B)(C)$ and compose binary matrices is associative.

Result: If $n=n_{1} n_{2} n_{3}$ and there are Hadmard matrices of orders $n_{1}, n_{2}, n_{3}$ then there is Hadmard matrix of order $n=n_{1} n_{2} n_{3}$.

Third. There is Identity for compose binary matrices that is Null matrix $O=[0]$ and for any binary matrix $A$ is $A(O)=O(A)=A$.

Forth. Compose binary matrices is not commutative:

\section{Example 6:}

$\left[\begin{array}{ll}0 & 1 \\ 1 & 0\end{array}\right]\left(\left[\begin{array}{lll}1 & 0 & 1 \\ 1 & 1 & 0 \\ 0 & 1 & 1\end{array}\right]\right)=\left[\begin{array}{lll|lll}1 & 0 & 1 & 0 & 1 & 0 \\ 1 & 1 & 0 & 0 & 0 & 1 \\ 0 & 1 & 1 & 1 & 0 & 0 \\ \hline 0 & 1 & 0 & 1 & 0 & 1 \\ 0 & 0 & 1 & 1 & 1 & 0 \\ 1 & 0 & 0 & 0 & 1 & 1\end{array}\right]$
And clear that:

$\left[\begin{array}{ll}0 & 1 \\ 1 & 0\end{array}\right]\left(\left[\begin{array}{lll}1 & 0 & 1 \\ 1 & 1 & 0 \\ 0 & 1 & 1\end{array}\right]\right) \neq\left[\begin{array}{lll}1 & 0 & 1 \\ 1 & 1 & 0 \\ 0 & 1 & 1\end{array}\right]\left(\left[\begin{array}{ll}0 & 1 \\ 1 & 0\end{array}\right]\right)$

Fifth. For the binary matrix $A$ there is no inverse $A^{\prime}$ such $A\left(A^{\prime}\right)=A^{\prime}(A)=O=[0]$.

Example 7: Suppose $(a)=a_{0}, a_{1}, a_{2}, \ldots, a_{n-1}$ and (b) $=b_{0}, b_{1}, b_{2}, \ldots, b_{m-1}$ are one period of two binary M-Sequences with the lengths $n=2^{k_{1}}-1$ , $m=2^{k_{2}}-1$ respectively, and the matrix $A$ is the all cyclic permutations of the sequence $(a)$, the matrix $B$ is the all cyclic permutation of the matrix (b).

Thus each row of the matrix $A$ contains $n_{1}=\left(2^{k_{1}-1}-1\right)$ of "0.s"and $n_{2}=2^{k_{1}-1}$ of " 1 .s".

The rows of the matrix $A$ are closed under the addition mod 2 and any two different rows are orthogonal and contain $f_{2}=2^{k_{1}-1}$ disagreements and $f_{1}=\left(2^{k_{1}-1}-1\right)$ of agreements, $\quad r_{1}=2^{k_{1}-2}$ of agreements are "1.s"and $r_{2}=\left(2^{k_{1}-2}-1\right)$ of agreements are "0.s".

Thus each row of the matrix $B$ contains $m_{1}=\left(2^{k_{2}-1}-1\right)$ of " $0 . s^{\prime}$ " and $m_{2}=2^{k_{2}-1}$ of "1.s".

The rows of the matrix $B$ are closed under the addition mod 2 and any two different rows are orthogonal and contain $g_{2}=2^{k_{2}-1}$ disagreements and $g_{1}=\left(2^{k_{2}-1}-1\right)$ of agreements, $h_{1}=2^{k_{2}-2}$ of agreements are "1.s" and $h_{2}=\left(2^{k_{2}-2}-1\right)$ of agreements are "0.s".

* In the composition $A(B)$ : 
- The length of each row is: Thus, second condition of the orthogonal is $n m=\left(2^{k_{1}}-1\right)\left(2^{k_{2}}-1\right)=2^{k_{1}+k_{2}}-\left(2^{k_{1}}+2^{k_{2}}\right)+1$ verified

- The number of "1.s" is:

is: For remove the non-orthogonal situations need $n_{1} m_{1}+n_{2} m_{2}=\left(2^{k_{1}-1}-1\right)\left(2^{k_{2}-1}-1\right)+2^{k_{1}-1} 2^{k_{2}-1}$ think about extend the rows of the matrix $A$ and the rows of the matrix $B$ by adding " 0 " at the beginning each row of them, thus we have:

$n_{1} m_{1}+n_{2} m_{2}=2^{k_{1}+k_{2}-1}-\left(2^{k_{1}-1}+2^{k_{2}-1}\right)+1$

- The number of "0.s" is: $n_{1} m_{2}+n_{2} m_{1}=\left(2^{k_{1}-1}-1\right) 2^{k_{2}-1}+2^{k_{1}-1}\left(2^{k_{2}-1}-1\right)$ $n_{1} m_{2}+n_{2} m_{1}=2^{k_{1}+k_{2}-1}-\left(2^{k_{1}-1}+2^{k_{2}-1}\right)$ $n=2^{k_{1}}, n_{1}=2^{k_{1}-1}, n_{2}=2^{k_{1}-1}, f_{1}=f_{2}=2^{k_{1}-1}$ $m=2^{k_{2}}, m_{1}=2^{k_{2}-1}, m_{2}=2^{k_{2}-1}, g_{1}=g_{2}=2^{k_{2}-1}$ * In the composition $\tilde{A}(\tilde{B})$ : where the " " is the signal of extending, and $\tilde{A}(\tilde{B})$ of the size $\left(2^{k_{1}}-1\right)\left(2^{k_{2}}-1\right) \times 2^{k_{1}} 2^{k_{2}}$. and the number of "1.s" is 1 . Thus, the first condition of the orthogonal is exist.

- In the composition $A_{i}\left(B_{j}\right)$ and $A_{i}\left(B_{k}\right)$ :

- The number of agreements is: $n g_{1}=\left(2^{k_{1}}-1\right)\left(2^{k_{2}-1}-1\right)=2^{k_{1}+k_{2}-1}-\left(2^{k_{1}}+2^{k_{2}-1}\right)+1$

- The length of each row is: $n m=2^{k_{1}} 2^{k_{2}}=2^{k_{1}+k_{2}}$

- The number of "1.s" is: $n_{1} m_{1}+n_{2} m_{2}=2^{k_{1}-1} 2^{k_{2}-1}+2^{k_{1}-1} 2^{k_{2}-1}=2^{k_{1}+k_{2}-1}$

- The number of "0.s" is:

- The number of disagreements is: is: $\quad n_{1} m_{2}+n_{2} m_{1}=2^{k_{1}-1} 2^{k_{2}-1}+2^{k_{1}-1} 2^{k_{2}-1}=2^{k_{1}+k_{2}-1}$ $n g_{2}=\left(2^{k_{1}}-1\right)\left(2^{k_{2}-1}\right)=2^{k_{1}+k_{2}-1}-2^{k_{2}-1}$.

- The difference $d$ between the

- The difference between the number of "0.s" and the number of " $1 . \mathrm{s"}$ " is 0 . disagreements and agreements is: $d=2^{k_{1}}-1$ Thus, second condition of the orthogonal is unverified.

* In the composition $A_{i}\left(B_{k}\right)$ and $A_{j}\left(B_{k}\right)$ :

- The number of agreements is: $m f_{1}=\left(2^{k_{2}}-1\right)\left(2^{k_{1}-1}-1\right)=2^{k_{1}+k_{2}-1}-\left(2^{k_{1}-1}+2^{k_{2}}\right)+1$

Thus, the first condition of the orthogonal is verified.

* In the composition $\widetilde{A}_{i}\left(\widetilde{B}_{j}\right)$ and $\tilde{A}_{i}\left(\tilde{B}_{k}\right)$ :

- The number of agreements is: $n g_{1}=2^{k_{1}} 2^{k_{2}-1}=2^{k_{1}+k_{2}-1}$.

- The number of disagreements is:

- The number of disagreements is: $m f_{2}=\left(2^{k_{2}}-1\right)\left(2^{k_{1}-1}\right)=2^{k_{1}+k_{2}-1}-2^{k_{1}-1}$.

- The difference $d$ between the disagreements and agreements is: $d=2^{k_{2}}-1$ Thus, second condition of the orthogonal is unverified.

* In the composition $A_{i}\left(B_{k}\right)$ and $A_{j}\left(B_{l}\right)$ :

- The number of agreements is: $f_{1} g_{1}+f_{2} g_{2}$.

- The number of disagreements is: $f_{1} g_{2}+f_{2} g_{1}$.

- The difference $d$ between the disagreements and agreements is:

$d=\left|\left(f_{1}-f_{2}\right)\left(g_{1}-g_{2}\right)\right|=\left|\left[\left(2^{k_{1}-1}-1\right)-2^{k_{1}-1}\right]\left[\left(2^{k_{2}-1}-1\right)-2^{k_{2}-1}\right]\right|=1$ $n g_{2}=2^{k_{1}} 2^{k_{2}-1}=2^{k_{1}+k_{2}-1}$.

- The difference $d$ between the disagreements and agreements is: $d=0$

Thus, second condition of the orthogonal is verified.

* In the composition $\tilde{A}_{i}\left(\tilde{B}_{k}\right)$ and $\tilde{A}_{j}\left(\tilde{B}_{k}\right)$ :

- The number of agreements is: $m f_{1}=2^{k_{2}} 2^{k_{1}-1}=2^{k_{1}+k_{2}-1}$.

- The number of disagreements is: $m f_{2}=2^{k_{2}} 2^{k_{1}-1}=2^{k_{1}+k_{2}-1}$.

- The difference $d$ between the disagreements and agreements is: $d=0$

Thus, second condition of the orthogonal is verified. 
* In the composition $\tilde{A}_{i}\left(\widetilde{B}_{k}\right)$ and $\tilde{A}_{j}\left(\tilde{B}_{l}\right)$ :

- The number of agreements is: $f_{1} g_{1}+f_{2} g_{2}$.

- The number of disagreements is: $f_{1} g_{2}+f_{2} g_{1}$.

From example 1. And example 2.:
- The difference $d$ between the disagreements and agreements is: $d=\left|\left(f_{1}-f_{2}\right)\left(g_{1}-g_{2}\right)\right|=0$

Thus, second condition of the orthogonal is verified.

$$
A=\left[\begin{array}{lll}
1 & 0 & 1 \\
1 & 1 & 0 \\
0 & 1 & 1
\end{array}\right], B=\left[\begin{array}{lllllll}
1 & 0 & 0 & 1 & 0 & 1 & 1 \\
1 & 1 & 0 & 0 & 1 & 0 & 1 \\
1 & 1 & 1 & 0 & 0 & 1 & 0 \\
0 & 1 & 1 & 1 & 0 & 0 & 1 \\
1 & 0 & 1 & 1 & 1 & 0 & 0 \\
0 & 1 & 0 & 1 & 1 & 1 & 0 \\
0 & 0 & 1 & 0 & 1 & 1 & 1
\end{array}\right], \bar{B}=\left[\begin{array}{lllllll}
0 & 1 & 1 & 0 & 1 & 0 & 0 \\
0 & 0 & 1 & 1 & 0 & 1 & 0 \\
0 & 0 & 0 & 1 & 1 & 0 & 1 \\
1 & 0 & 0 & 0 & 1 & 1 & 0 \\
0 & 1 & 0 & 0 & 0 & 1 & 1 \\
1 & 0 & 1 & 0 & 0 & 0 & 1 \\
1 & 1 & 0 & 1 & 0 & 0 & 0
\end{array}\right]
$$

And

$A(B)=\left[\begin{array}{lllllll|lllllll|lllllll}0 & 1 & 1 & 0 & 1 & 0 & 0 & 1 & 0 & 0 & 1 & 0 & 1 & 1 & 0 & 1 & 1 & 0 & 1 & 0 & 0 \\ 0 & 0 & 1 & 1 & 0 & 1 & 0 & 1 & 1 & 0 & 0 & 1 & 0 & 1 & 0 & 0 & 1 & 1 & 0 & 1 & 0 \\ 0 & 0 & 0 & 1 & 1 & 0 & 1 & 1 & 1 & 1 & 0 & 0 & 1 & 0 & 0 & 0 & 0 & 1 & 1 & 0 & 1 \\ 1 & 0 & 0 & 0 & 1 & 1 & 0 & 0 & 1 & 1 & 1 & 0 & 0 & 1 & 1 & 0 & 0 & 0 & 1 & 1 & 0 \\ 0 & 1 & 0 & 0 & 0 & 1 & 1 & 1 & 0 & 1 & 1 & 1 & 0 & 0 & 0 & 1 & 0 & 0 & 0 & 1 & 1 \\ 1 & 0 & 1 & 0 & 0 & 0 & 1 & 0 & 1 & 0 & 1 & 1 & 1 & 0 & 1 & 0 & 1 & 0 & 0 & 0 & 1 \\ 1 & 1 & 0 & 1 & 0 & 0 & 0 & 0 & 0 & 1 & 0 & 1 & 1 & 1 & 1 & 1 & 0 & 1 & 0 & 0 & 0 \\ \hline 0 & 1 & 1 & 0 & 1 & 0 & 0 & 0 & 1 & 1 & 0 & 1 & 0 & 0 & 1 & 0 & 0 & 1 & 0 & 1 & 1 \\ 0 & 0 & 1 & 1 & 0 & 1 & 0 & 0 & 0 & 1 & 1 & 0 & 1 & 0 & 1 & 1 & 0 & 0 & 1 & 0 & 1 \\ 0 & 0 & 0 & 1 & 1 & 0 & 1 & 0 & 0 & 0 & 1 & 1 & 0 & 1 & 1 & 1 & 1 & 0 & 0 & 1 & 0 \\ 1 & 0 & 0 & 0 & 1 & 1 & 0 & 1 & 0 & 0 & 0 & 1 & 1 & 0 & 0 & 1 & 1 & 1 & 0 & 0 & 1 \\ 0 & 1 & 0 & 0 & 0 & 1 & 1 & 0 & 1 & 0 & 0 & 0 & 1 & 1 & 1 & 0 & 1 & 1 & 1 & 0 & 0 \\ 1 & 0 & 1 & 0 & 0 & 0 & 1 & 1 & 0 & 1 & 0 & 0 & 0 & 1 & 0 & 1 & 0 & 1 & 1 & 1 & 0 \\ 1 & 1 & 0 & 1 & 0 & 0 & 0 & 1 & 1 & 0 & 1 & 0 & 0 & 0 & 0 & 0 & 1 & 0 & 1 & 1 & 1 \\ 1 & 0 & 0 & 1 & 0 & 1 & 1 & 0 & 1 & 1 & 0 & 1 & 0 & 0 & 0 & 1 & 1 & 0 & 1 & 0 & 0 \\ 1 & 1 & 0 & 0 & 1 & 0 & 1 & 0 & 0 & 1 & 1 & 0 & 1 & 0 & 0 & 0 & 1 & 1 & 0 & 1 & 0 \\ 1 & 1 & 1 & 0 & 0 & 1 & 0 & 0 & 0 & 0 & 1 & 1 & 0 & 1 & 0 & 0 & 0 & 1 & 1 & 0 & 1 \\ 0 & 1 & 1 & 1 & 0 & 0 & 1 & 1 & 0 & 0 & 0 & 1 & 1 & 0 & 1 & 0 & 0 & 0 & 1 & 1 & 0 \\ 1 & 0 & 1 & 1 & 1 & 0 & 0 & 0 & 1 & 0 & 0 & 0 & 1 & 1 & 0 & 1 & 0 & 0 & 0 & 1 & 1 \\ 0 & 1 & 0 & 1 & 1 & 1 & 0 & 1 & 0 & 1 & 0 & 0 & 0 & 1 & 1 & 0 & 1 & 0 & 0 & 0 & 1 \\ 0 & 0 & 1 & 0 & 1 & 1 & 1 & 1 & 1 & 0 & 1 & 0 & 0 & 0 & 1 & 1 & 0 & 1 & 0 & 0 & 0\end{array}\right]$

* In the composition $A(B)$ :

Thus, the first condition of the orthogonal is

- The length of each row is: exist.

$n m=\left(2^{k_{1}}-1\right)\left(2^{k_{2}}-1\right)=3(7)=21$

* In the composition $A_{i}\left(B_{j}\right)$ and $A_{i}\left(B_{k}\right)$ :

- The number of "1.s" $n_{1} m_{1}+n_{2} m_{2}=1(3)+2(4)=11$

- The number of "0.s" $n_{1} m_{2}+n_{2} m_{1}=1(4)+2(3)=10$

- The difference between the number of " $0 . \mathrm{s}$ " and the number of " $1 . \mathrm{s}$ " is 1 .

is: - The number of agreements is: $n g_{1}=3(3)=9$

- The number of disagreements is: is: $\quad n g_{2}=3(4)=12$.

- The difference $d$ between the disagreements and agreements is: $d=3$. 
For example, the first seven rows, or between $\quad$ In the composition $A_{i}\left(B_{k}\right)$ and $A_{j}\left(B_{l}\right)$ :

8-14 rows, or between 15-21 rows. Thus, second condition of the orthogonal is unverified.

* In the composition $A_{i}\left(B_{k}\right)$ and $A_{j}\left(B_{k}\right)$ :

- The number of agreements $m f_{1}=7(1)=7$.

- The number of disagreements is: $m f_{2}=7(2)=14$.

- The difference $d$ between the disagreements and agreements is: $d=7$.

For examples: between the $1^{\text {th }}$ row and $8^{\text {th }}$ row, between $2^{\text {th }}$ row and $9^{\text {th }}$ row, ... Thus, second condition of the orthogonal is unverified.

is:
- The number of disagreements

is:

$$
f_{1} g_{2}+f_{2} g_{1}=1(4)+2(3)=10 \text {. }
$$

- The difference $d$ between the disagreements and agreements is:

$$
d=\left|\left(f_{1}-f_{2}\right)\left(g_{1}-g_{2}\right)\right|=\left|\left[\left(2^{k_{1}-1}-1\right)-2^{k_{1}-1}\right]\left[\left(2^{k_{2}-1}-1\right)-2^{k_{2}-1}\right]\right|=1
$$

For example: between the $1^{\text {th }}$ and $9^{\text {th }}$ row, between $1^{\text {th }}$ row and $10^{\text {th }}$ row, ..., second condition of the orthogonal is verified.

By extend the matrices $A$ and $B$ :

$$
\tilde{A}=\left[\begin{array}{llll}
0 & 1 & 0 & 1 \\
0 & 1 & 1 & 0 \\
0 & 0 & 1 & 1
\end{array}\right], \tilde{B}=\left[\begin{array}{llllllll}
0 & 1 & 0 & 0 & 1 & 0 & 1 & 1 \\
0 & 1 & 1 & 0 & 0 & 1 & 0 & 1 \\
0 & 1 & 1 & 1 & 0 & 0 & 1 & 0 \\
0 & 0 & 1 & 1 & 1 & 0 & 0 & 1 \\
0 & 1 & 0 & 1 & 1 & 1 & 0 & 0 \\
0 & 0 & 1 & 0 & 1 & 1 & 1 & 0 \\
0 & 0 & 0 & 1 & 0 & 1 & 1 & 1
\end{array}\right], \quad \tilde{\widetilde{B}}=\left[\begin{array}{llllllll}
1 & 0 & 1 & 1 & 0 & 1 & 0 & 0 \\
1 & 0 & 0 & 1 & 1 & 0 & 1 & 0 \\
1 & 0 & 0 & 0 & 1 & 1 & 0 & 1 \\
1 & 1 & 0 & 0 & 0 & 1 & 1 & 0 \\
1 & 0 & 1 & 0 & 0 & 0 & 1 & 1 \\
1 & 1 & 0 & 1 & 0 & 0 & 0 & 1 \\
1 & 1 & 1 & 0 & 1 & 0 & 0 & 0
\end{array}\right]
$$

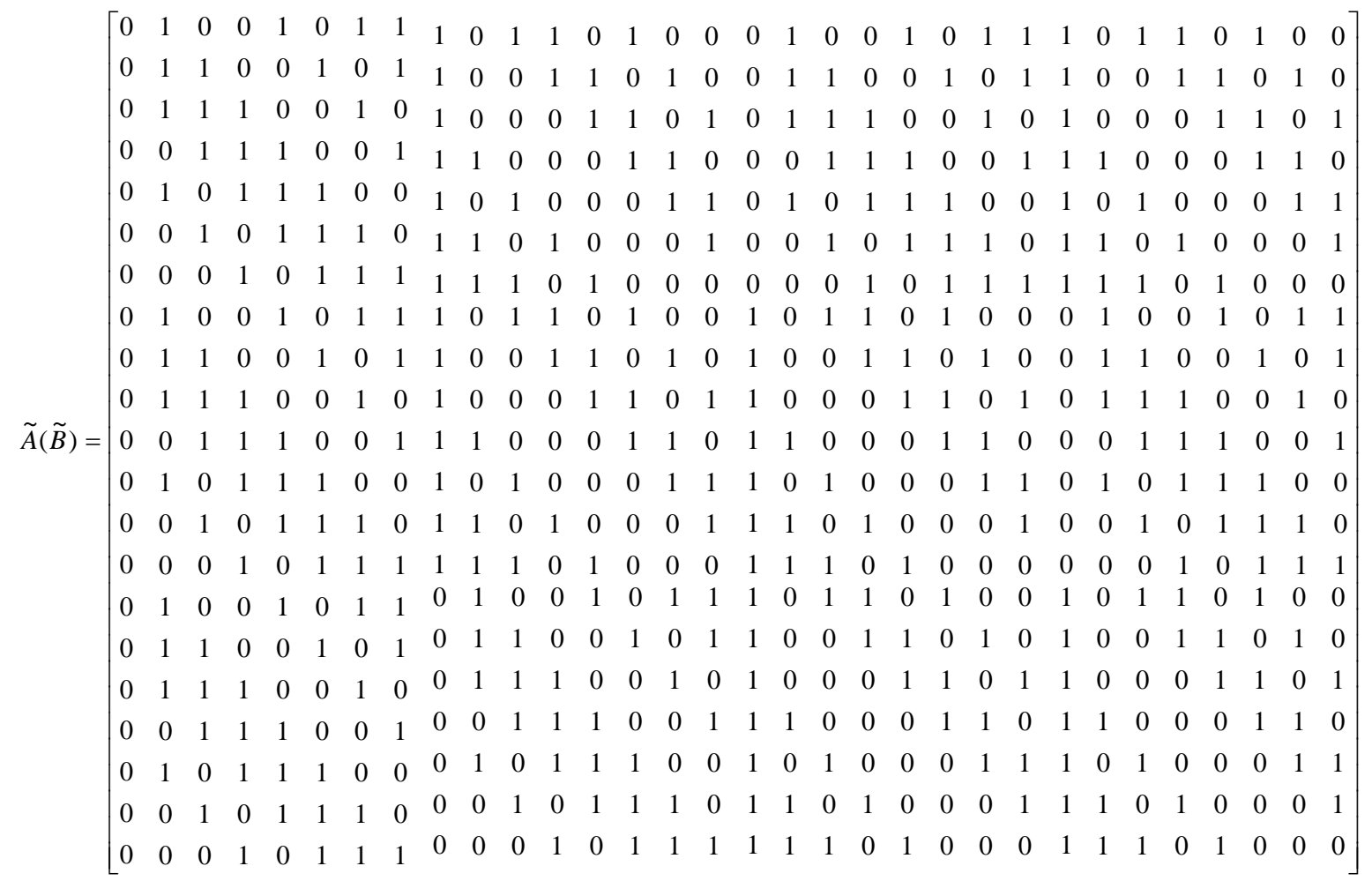

The length of any row is: $4(8)=32$ and contains 16 of " $0 . s$ " and 16 of "1.s". Any two different row contain 16 of agreements and 16 of disagreements and rows of $\tilde{A}(\tilde{B})$ is closed under the addition by mod 2 . Thus rows of $\tilde{A}(\tilde{B})$ is an orthogonal set. Coming back to system $\{1,-1\}$ replacing each " 1 " by " 1 " and each " 0 " by " 1 ". 


\section{Conclusion}

1. The operation of compose binary matrices is associative, non-commutative, there is identity and no inverse.

2. compose two Hadamard matrices is also Hadamard matrix.

3. If $n=n_{1} \cdot n_{2} \ldots . n_{k}$ and there are Hadamard matrices of orders $n_{1}, n_{2}, \ldots, n_{k}$ then there is Hadamard matrix of order $n$

4. Using compose matrices we can get Hadamard matrices with the bigger lengths and the bigger minimum distance that assists to increase secrecy of these information and increase the possibility of correcting mistakes resulting in the channels of communication.

5. Compose two matrices of M-Sequences is not matrix of M-Sequences and the result of composition is not orthogonal set.

6. we can extend the matrices of M-Sequences to getting orthogonal matrix with big size, with

the bigger lengths and the bigger minimum distance that assists to increase secrecy of these information and increase the possibility of correcting mistakes resulting in the channels of communication.

\section{Acknowledgment}

The author expresses their gratitude to Prof. Abdulla Y Al Hawaj, President of Ahlia University for all the Support.

\section{References}

[1] Al Cheikha, A. H. (2014), Composed Short Walsh's Sequences, American International Journal for Contemporary Scientific Research, 1(2), 81-88.

[2] Al Cheikha, A. H. (2005), Generation of sets of sequences isomorphic to Walsh sequences. Qatar University Science Journal, 25, 16-30.

[3] Byrnes, J.S.; Swick,(1970), Instant Walsh Functions, SIAM Review., Vol. 12, pp.131.

[4] Brouwer, A. E.; Cohen, A. M.; and Neumaier, A.(1989), "Hadamard Matrices" $\$ 1.8$ in Distance Regular Graphs. New York: Springer-Verlag, pp. 19-20,.
[5] Djoković, D. Z. (2009),"Hadamard Matrices of Small Order and Yang Conjecture" http://arxiv.org/abs/0912.5091.

[6] Evangelaras, H.; Koukouvinos C.; Seberry J.(2003), applications of Hadamard matrices, Journal of telecommunication and information technology. Pp. 3-10

[7] Fraleigh, J. B. (1971), A First course In Abstract Algebra. Fourth printing, USA: Addison-Wesley publishing company.

[8] Geramita,A.V., Seberry, J.(1979), Orthogonal designs, quadratic forms and Hadamard Matrices, Lecture Notes in Pure and Applied Mathematics, vol.43, Marcel Dekker, NewYork and Basel.

[9] Geramita, A.V., Seberry, J.(1979), Orthogonal Designs: Quadratic Forms and Hadamard Matrices, New York-Basel: Marcel Dekker.

[10] Hedayat, A.S., Sloane, N.J.A., Stufken, J.(1999), Orthogonal arrays theory and Applications, SpringerVerlag, New York.

[11] Hedayat, A., Wallis, W.D.(1978), Hadamard matrices and their applications. Ann. Stat. 6, 1184-1238

[12] Jong-Seon No, Solomon W. \& Golomb,(1998), Binary Pseudorandom Sequences For period $2^{\mathrm{n}}-1$ with Ideal Autocorrelation, IEEE Trans. Information Theory, Vol. 44 No 2, PP 814-817

[13] Kitis, L. "Paley's Construction of Hadamard.

[14] Koukouvinos, C.; Kounias, S.(1998), An infinite class of Hadamard matrices. J Austral SocA 46, 384-394 18 Seberry et al.

[15] Lee, J. S., Miller. L. E. (1998 ), CDMA Systems Engineering Handbook. Boston, London: Artech House.

[16] Lidl, R.\& Nidereiter, H.,(1994), Introduction to Finite Fields and Their Application, Cambridge University USA.

[17] Lidl, R.\& Pilz,G., "Applied Abstract Algebra," Springer-VerlageNew York, 1984

[18] Mac Williams, F. J.; Sloane, N. J. A. (2006), The theory of Error- correcting Codes, Amsterdam: NorthHolland Publishing Company

[19] Seberry, J. (2004), Library of hadamard matrices, http//www.uow.edu.au/ jennie/ hadamard.html.

[20] Seberry, J., Yamada, M.,(1992), Hadamard matrices, sequences, and block designs, In: Dinitz $\mathrm{JH}$, Stinson DR (eds) Contemporary design theory: a collection of surveys, JohnWiley \& Sons, Inc., Pp 431437.

[21] Seberry, J.; Wysocki, B.J.; Wysocki, T.A.,(2003) Williamson-Hadamard spreading Sequences for DSCDMA applications. J.Wireless Commun. Mobile Comput, 3(5), 597-607.

[22] Seberry, J.; JWysocki, B. ; AWysocki, T., On some applications of Hadamard matrices.

[23] Seberry, J.; Wysocki, B.J.; Wysocki, T.A.; Tran, L.C.; Wang, Y.; Xia, T.; Zhao, Y., (2004), Complex orthogonal sequences from amicable Hadamard matrices, IEEE 
VTC' Spring, Milan, Italy, 17-19 May 2004 - CD ROM, 2004

[24] Seberry J., Yamada M.,(1992), Hadamard matrices, sequences and designs, in Design Theory - a Collection of Surveys, D. J. Stinson and J. Dinitz, Eds. Wiley, Pp. 431-560.

[25] Seberry J.; Wallis, (1972), Part IV of combinatorics: Room squares, sum free sets and Hadamard matrices, Lecture Notes in Mathematics, W. D. Wallis, A. Pen fold Street, and J. Seberry Wallis, Eds. Berlin- HeidelbergNew York: Springer, vol. 292.

[26] Sloane, N.J.A.(2004), A library of Hadamard matrices, http//www.research.att.com/ najs/hadamard/.

[27] Sloane, N.J.A., (1976), An Analysis Of The Stricture and Complexity Of Nonlinear Binary Sequence Generators, IEEE Trans. Information Theory Vol. It 22 No 6,PP 732-736.

[28] Thomson W. Judson, (2013), Abstract Algebra: Theory and Applications, Free Software Foundation.

[29] Wolfram Notebook, Hadamard Matrix.

[30] Wysocki, B.J.; Wysocki, T.A., (2002), Modified Walsh-Hadamard sequences for DS CDMA wireless systems. Int. J. Adapt. Control Signal Process., 16589 602.

[31] Yang; Samuel C., (1998), CDMA RF Engineering. Artech House, Boston London.

[32] Yarlagadda, R.K.; Hershey, J.E.:(1997), Hadamard matrix analysis and synthesis with applications to communications and signal/image processing. Kluwer. 